\title{
OPTIMIZATION OF DROPLET DIGITAL POLYMERASE CHAIN REACTION (ddPCR) FOR DNA COPY NUMBER VARIATION ANALYSIS OF CNV ESV27061 IN YOUNG ADULTS WITH HIGHER BLOOD PRESSURE
}

\author{
Siti Radziah Shaik Alaudeen ${ }^{1}$, Aszrin Abdullah ${ }^{1}$, Azarisman Shah Mohd Shah ${ }^{2}$ and \\ Norlelawati Abdul Talib ${ }^{3}$ \\ ${ }^{1}$ Department of Basic Medical Sciences, Kulliyyah of Medicine, International Islamic \\ University Malaysia, Pahang, Malaysia, ${ }^{2}$ Department of Internal Medicine, Kulliyyah of \\ Medicine, International Islamic University Malaysia, Pahang, Malaysia, ${ }^{3}$ Department of \\ Pathology and Laboratory Medicine, Kulliyyah of Medicine, International Islamic \\ University Malaysia, Pahang, Malaysia
}

Presenter: Siti Radziah, sitiradziah@yahoo.com

Introduction: Copy number variation (CNV) caused by changes in DNA sequences of 1000 or more bases is implicated with susceptibility to common diseases. A study on CNV esv27061 among hypertensive Australian adults reported association with high blood pressure (BP). In Malaysia, no study on CNV among hypertensive young adults is available. Thus, this investigation aimed to assess the CNV esv27061 of young Malaysian adults with high blood pressure using optimized ddPCR.

Materials and method: Ten samples each from hypertensive and healthy controls were randomly selected from samples collected for an on-going comparative cross-sectional research project among young adults living in Kuantan. The DNAs were purified using Maxwell RSC Buffy Coat DNA Kit and the concentration was measured using SimpliNano spectrophotometer. Subsequently, restriction digestion of DNAs by EcoRV was performed prior to ddPCR. The products were later subjected to droplet generation (QX100 Droplet Generator), PCR amplification and finally CNV was read by QX100 Droplet reader. Unfortunately, the above method did not yield any result. Thus, an alternative method in which purified DNA concentration was determined by QuantiFluor ONE dsDNA System (Quantus fluorometer). The DNAs (60 ng) and Alu1 were added in master mix during ddPCR and CNV esv27061 analysis was performed as stated above.

Results: Optimization of method in this study showed that the detection of CNV esv27061 was possible by the use of more sensitive measurement of DNA concentration, Alu1 restriction enzyme instead of EcoRV and digestion in ddPCR reaction method rather than prior digestion. The finalized protocol run on selected hypertensive and healthy controls has shown to be reproducible and easily interpretable discrimination of gene's copy numbers.

Conclusion: This optimized protocol for CNV esv27061 analysis proved useful in identifying CNV and will allow a reproducible assay evaluation and the application of this method to a bigger sample size. 